ADP-03-126/T562

\title{
Observing Chiral Nonanalytic Behavior with FLIC Fermions
}

\author{
D. B. Leinweber ${ }^{\mathrm{a}}$, A. W. Thomas ${ }^{\mathrm{a}}$, A. G. Williams ${ }^{\mathrm{a}}$, R. D. Young ${ }^{\mathrm{a}}$, J. M. Zanotti ${ }^{\mathrm{ab}}$ and \\ J. B. Zhanga

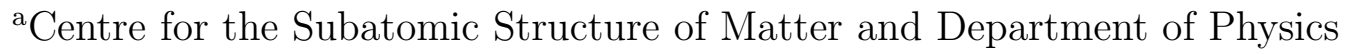 \\ University of Adelaide, Adelaide SA 5005 Australia \\ b John von Neumann-Institut für Computing NIC, \\ Deutches Elektronen-Synchrotron DESY, D-15738 Zeuthen, Germany
}

First results from lattice QCD revealing the chiral nonanalytic behavior of nucleon and $\Delta$ baryon magnetic moments are presented. Numerical simulations in the light quark mass regime employing the nonperturbatively $\mathcal{O}(a)$-improved conserved vector current are enabled via FLIC fermions. Quenched chiral perturbation theory for the nucleon and $\Delta$ magnetic moments is derived to next to next to leading nonanalytic order. Numerical simulation results for the proton and $\Delta$ baryon magnetic moments in quenched QCD reveal dramatic signatures of the quenched meson cloud, which are in accord with the predictions of quenched chiral perturbation theory.

\section{NUMERICAL SIMULATIONS WITH FLIC FERMIONS}

Access to leading-edge supercomputing resources coupled with advances in the formulation of computationally-inexpensive chirally-improved lattice fermion actions [ 1, 2] enable the numerical calculation of hadron structure in the chiral regime. In this regime, the pseudo-Goldstone boson dressings of hadrons give rise to significant non-analytic curvature in the quark-mass dependence of observables. The magnetic moments of baryons have been identified [ [3, 4] as providing an excellent opportunity for the direct observation of chiral nonanalytic behavior in lattice QCD, even in the quenched approximation.

The numerical simulations of the electromagnetic form factors presented here are carried out using the Fat Link Irrelevant Clover (FLIC) fermion action [ 1, 2] in which the irrelevant operators introduced to remove fermion doublers and lattice spacing artifacts are constructed with smoothed links. These links are created via APE smearing [ [5]; a process that averages a link with its nearest transverse neighbors in a gauge invariant manner. Iteration of the averaging process generates a "fat" link.

The use of links in which short-distance fluctuations have been removed simplifies the determination of the coefficients of the improvement terms in both the action and its associated conserved vector current. Perturbative renormalizations are small for smeared links and the mean-field improved coefficients used here are sufficient to remove $\mathcal{O}(a)$ errors, in the lattice spacing $a$, from the lattice fermion action. The key is that both the energy dimension-five Wilson and Clover terms [ 6] are constructed with smooth links, 
while the relevant operators, surviving in the continuum limit, are constructed with the original untouched links generated via standard Monte Carlo techniques.

FLIC fermions provide a new form of nonperturbative $\mathcal{O}(a)$ improvement [ 2, 7] where near-continuum results are obtained at finite lattice spacing. Access to the light quark mass regime is enabled by the improved chiral properties of the lattice fermion action. The magnitude of additive mass renormalizations is suppressed [ 7] which otherwise can lead to singular behavior in the propagators as the quarks become light.

The $\mathcal{O}(a)$-improved conserved vector current [8] is used. Nonperturbative improvement is achieved via the FLIC procedure where the terms of the Noether current having their origin in the irrelevant operators of the fermion action are constructed with mean-field improved APE smeared links. The preliminary results presented here are from a sample of $25520^{3} \times 40$ mean-field improved Luscher-Weisz [9] gauge field configurations having a lattice spacing of $0.128 \mathrm{fm}$ as determined by the Sommer scale $r_{0}=0.50 \mathrm{fm}$.

\section{CHIRAL NONANALYTIC BEHAVIOR}

The truncation of the low-energy expansion of chiral effective field theory introduces errors into the predictions of chiral perturbation theory $(\chi \mathrm{PT})$. In the process of a simple truncation, one sets the coefficients of higher-order terms of the expansion (both analytic and nonanalytic) to zero by hand. While such a procedure is often described as "systematic" or "model independent" the truth is that the coefficients of these higher-order terms are generally not zero. Thus, the truncated expansion is a poor representation of the chiral expansion of QCD. In this case, "model independent" simply means that no attempt has been made to estimate the coefficients of higher order terms in the expansion. It is not necessarily a good feature.

While such an approach might be forgiven if the coefficients of the higher order terms were indeed small, there is now mounting evidence that this is not the case. For the nucleon mass, the best determination of the low-energy constants [10] from the physical nucleon mass and state-of-the-art lattice QCD results [11] indicate the nucleon mass has the following chiral expansion (in appropriate powers of $\mathrm{GeV}$ )

$$
\begin{aligned}
m_{N}= & (0.897 \pm 0.001)+(2.84 \pm 0.04) m_{\pi}^{2}+\chi_{3} m_{\pi}^{3}+(22.0 \pm 1.6) m_{\pi}^{4} \\
& +\chi_{4} m_{\pi}^{4} \log \left(m_{\pi}^{2} / 1 \mathrm{GeV}^{2}\right)+\chi_{5} m_{\pi}^{5}+\cdots,
\end{aligned}
$$

where $\chi_{i}$ are the known model-independent coefficients of the leading nonanalytic terms of the expansion and the quoted uncertainties are purely systematic [10]. The coefficient

of the $m_{\pi}^{6}$ term from the $N \rightarrow N \pi$ self energy alone is $-75 \pm 35 \mathrm{GeV}^{-5}$. Hence the "systematic" approach of setting the coefficients of all higher order terms to zero can be troublesome for the power-series like expansion of dimensional-regularization (DR).

Fortunately there is a way to estimate the coefficients of the higher order terms, while preserving the model-independent features of chiral effective field theory to the chiralcounting order that one is working. Through the process of regulating loop integrals via a finite-range regulator (FRR) [10], one re-sums the chiral expansion in a manner which preserves the model-independent features of chiral perturbation theory. For example, the coefficients of the leading nonanalytic terms of the expansion are preserved exactly. However, in the process of expanding the expressions of $\mathrm{FRR} \chi \mathrm{PT}$ to recover the expansion 

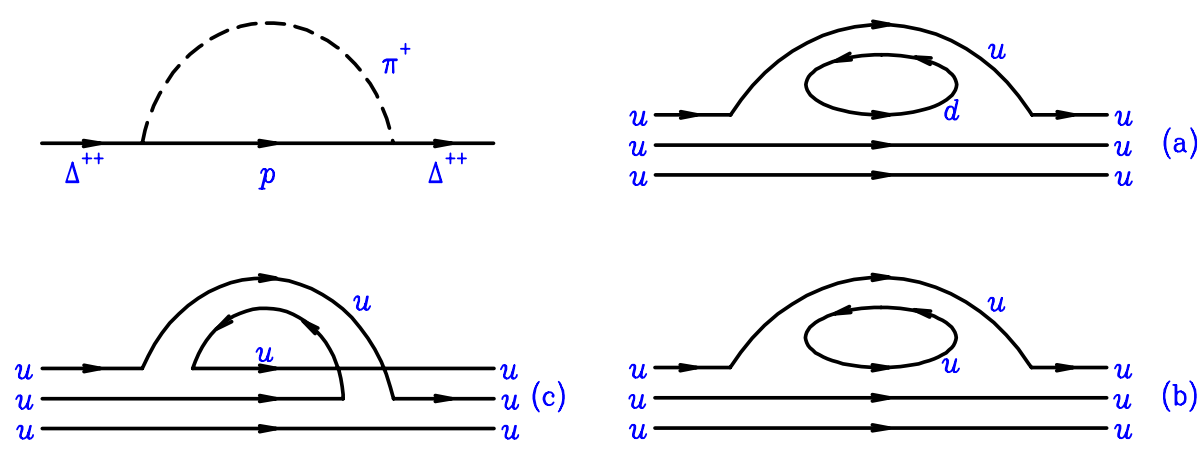

$(\mathrm{c})=-(\mathrm{b})=-(\mathrm{a})$

$(\mathrm{b})=(\mathrm{a})$

Figure 1. Quark-flow diagrams for meson-cloud contributions to $\Delta^{++}$in full QCD.

of DR in Eq. (11), one also encounters higher-order terms of the chiral expansion, whose coefficients are functions of the regulator parameter $(\Lambda)$ governing the finite range of the regulator. By optimizing $\Lambda$ in a fit of the FRR expansion to lattice QCD data, one obtains estimates for the higher order terms of the chiral expansion, while maintaining complete model-independence to the chiral order one is working.

The introduction of a finite range regulator opens the question of the functional form of the regulator. Clearly one needs a functional form that preserves the low energy physics of the chiral expansion, while suppressing high-energy contributions where the internal structure of the effective fields becomes important. In practice, sharp-cutoff, monopole, dipole and Gaussian vertex regulators have been investigated in some detail. Provided one allows the regulator parameter to be constrained by lattice QCD data, a remarkable robustness is observed in the predictions of the chiral expansion. Systematic errors for the nucleon mass have been estimated at less than 1\%, provided a smooth FRR (monopole, dipole, Gaussian) is selected [10]. These regulators not only provide estimates for the coefficients of higher-order analytic terms of the expansion, but also provide estimates for the coefficients of non-analytic terms which must also appear in the expansion. This latter feature of the smooth regulators has been identified as key to the success of the smooth regulators over the sharp cutoff [10].

We use the diagrammatic method for evaluating the quenched chiral coefficients of leading nonanalytic terms in heavy-baryon quenched $\chi \mathrm{PT}[$ [3, 4]. Results for the proton magnetic moment to next to next to leading nonanalytic (NNLNA) order [ 4, 12] are generalized to the FRR approach used here. In quenched QCD, the $\Delta$ form factors are simply proportional to the charge of the baryon [13]. Hence, consideration of the $\Delta^{++}$ charge state is sufficient to determine the chiral expansion for all charge states.

The presence of the $\Delta \rightarrow N \pi$ decay channel is particularly important for the mass dependence of $\Delta$ properties. Rapid curvature associated with nonanalytic behavior is shifted to larger pion masses near the $N-\Delta$ mass splitting, $m_{\pi} \sim M_{\Delta}-M_{N}$. As described below, quenched-QCD decay-channel contributions come with a sign opposite to that of full QCD. This artifact holds tremendous promise for revealing unmistakable signatures of the quenched meson cloud.

The change in sign for the decay-channel contributions is easily understood through the consideration of the quark flow diagrams of Fig. 1, illustrating the meson-cloud contributions to the $\Delta^{++}$resonance in full QCD. Quark flow diagram (a) corresponds to 


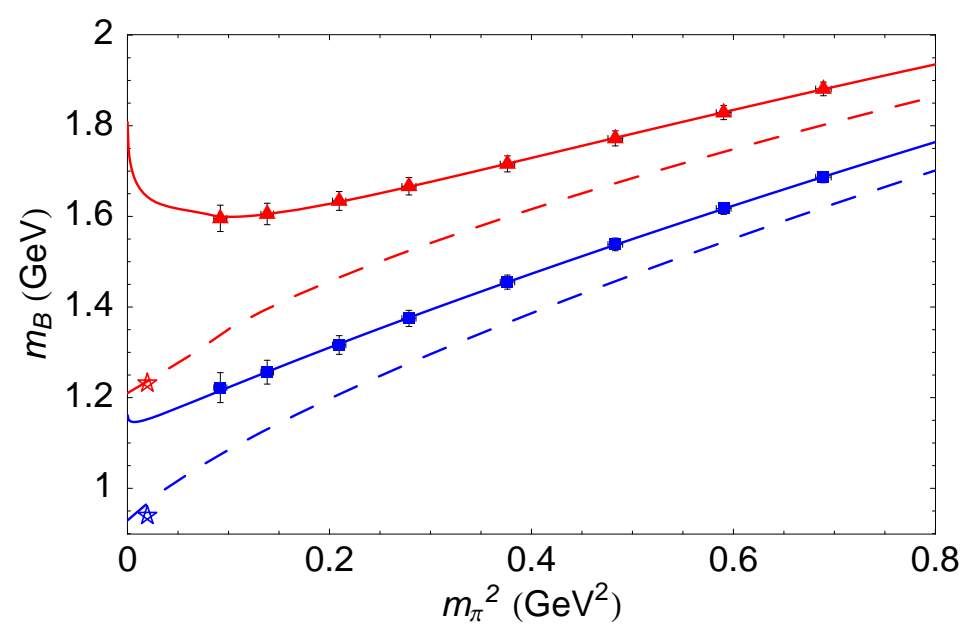

Figure 2. FLIC fermion simulation results for the masses of the proton (o) and $\Delta$ resonance $(\triangle)$ in quenched QCD. Solid curves indicate the fits of FRR quenched $\chi \mathrm{PT}$ to the lattice simulation results, while dashed curves indicate the one-loop correction to the quenched approximation [15]. Stars denote the physical values.

the hadronic process described at left. Since QCD is flavor-blind, the process illustrated in diagram (b) is equivalent to diagram (a) provided the masses of the $u$ and $d$ quarks are taken to be equal. On its own, diagram (b) describes the decay of the $\Delta^{++}$to a doubly-charged uuu "proton," which we denote $p^{++}$. Of course, such states do not exist in full QCD and diagram (c) makes contributions exactly equal but opposite in sign to diagram (b) when the intermediate state is a uuu proton. Upon quenching the theory, both diagrams (a) and (b) are eliminated, leaving only diagram (c). Hence the physics of the $\Delta \rightarrow N \pi$ decay is present in the quenched approximation [14] but its contribution has the wrong sign.

This aspect of the quenched $\Delta$ is the predominant feature giving rise to the flattening of the $\Delta$ mass as a function of quark mass at the lightest FLIC-fermion quark masses depicted in Fig. 2. Double-hairpin $\eta^{\prime}$ contributions impact the $\Delta$ mass at much lighter quark masses most notably between the chiral and physical pion mass.

As for the proton, the double-hairpin $\eta^{\prime}$ dressing $\Delta^{++} \rightarrow \Delta^{++} \eta^{\prime}$ provides the LNA contribution to the $\Delta^{++}$magnetic moment, generating a logarithmic divergence in the chiral limit. For electromagnetic form factors, Fig. 1(c) indicates that the coefficient of the NLNA contribution proportional to $m_{\pi}$ will vanish in the quenched approximation because of the neutral charge of the meson. However, Fig. 1(c) will make significant contributions when the electromagnetic current couples to the intermediate $p^{++}$. We estimate the tree-level magnetic moment of the uuu proton using standard $\mathrm{SU}(6)$ symmetry, $\mu_{p^{++}}=$ $\frac{4}{3} \mu_{u}-\frac{1}{3} \mu_{u}=\frac{2}{3} \mu_{p}=\frac{1}{3} \mu_{\Delta^{++}}$. The quark flow diagrams of Fig. 1 also include contributions from $\Delta$ intermediate states. In terms of the full QCD process $\Delta^{++} \rightarrow \Delta^{++} \pi^{0}$ we find the total quenched contribution to be $(4 / 3)\left(\Delta^{++} \rightarrow \Delta^{++} \pi^{0}\right)$.

Figure 3 displays FLIC fermion simulation results for the magnetic moments of the proton and $\Delta^{+}$resonance in quenched QCD. The curves illustrate the fits of FRR quenched $\chi \mathrm{PT}$ to the lattice simulation results. Here, the analytic terms of the chiral expansion have been re-summed in a Padé designed to reproduce the Dirac moment mass dependence, $e \hbar / 2 m$, at moderately large pion mass. 


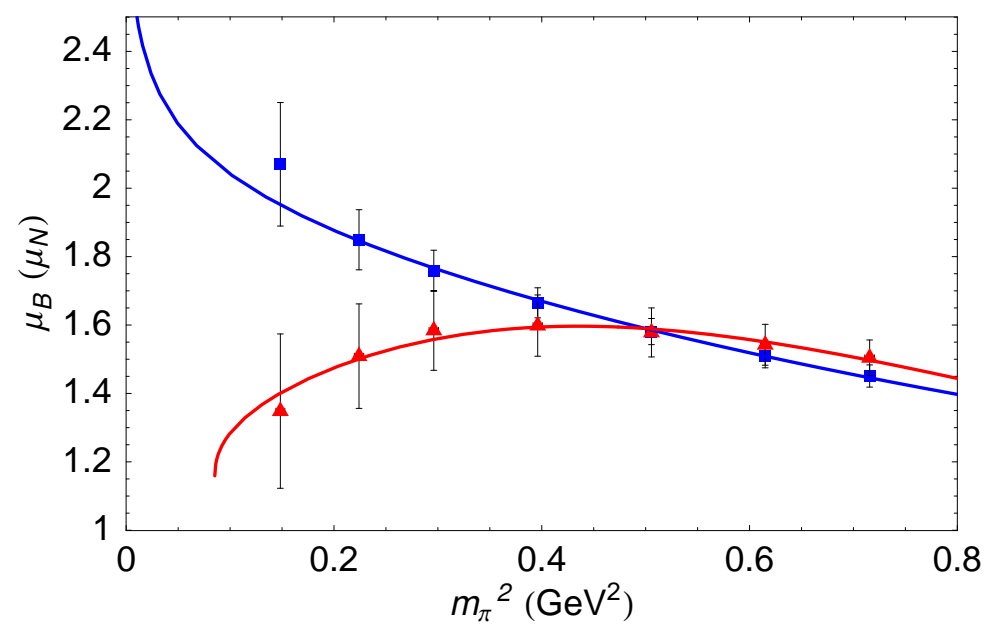

Figure 3. FLIC fermion simulation results for the magnetic moments of the proton (o) and $\Delta^{+}$resonance $(\triangle)$ in quenched QCD and the associated fits of FRR quenched $\chi \mathrm{PT}$ to the lattice simulation results.

At large pion masses, the $\Delta$ moment is enhanced relative to the proton moment in accord with earlier lattice QCD results [16, 17] and model expectations. However as the chiral regime is approached the nonanalytic behavior of the quenched meson cloud is revealed, enhancing the proton and suppressing the $\Delta^{+}$in accord with the expectations of quenched $\chi \mathrm{PT}$. The quenched artifacts of the $\Delta$ provide an unmistakable signal for the onset of quenched chiral nonanalytic behavior.

Supercomputing resources from the Australian Partnership for Advanced Computing and research support from the Australian Research Council is gratefully acknowledged.

\section{REFERENCES}

1. J. M. Zanotti et al. [CSSM Lattice Collaboration], Phys. Rev. D 65 (2002) 074507 arXiv:hep-lat/0110216.

2. D. B. Leinweber et al., arXiv:nucl-th/0211014.

3. D. B. Leinweber, Nucl. Phys. Proc. Suppl. 109 (2002) 45 arXiv:hep-lat/0112021.

4. D. B. Leinweber, arXiv:hep-lat/0211017.

5. M. Falcioni et al., Nucl. Phys. B251, 624 (1985).

6. S. O. Bilson-Thompson et al., Annals Phys. 304 (2003) 1 arXiv:hep-lat/0203008.

7. J. Zanotti, et al., in preparation.

8. G. Martinelli, C. T. Sachrajda and A. Vladikas, Nucl. Phys. B 358 (1991) 212.

9. M. Luscher and P. Weisz, Commun. Math. Phys. 97, 59 (1985) [ibid. 98, 433 (1985)].

10. R. D. Young et al., Prog. Part. Nucl. Phys. 50 (2003) 399 arXiv:hep-lat/0212031.

11. CP-PACS, A. Ali Khan et al., Phys. Rev. D65 (2002) 054505, hep-lat/0105015.

12. M. J. Savage, Nucl. Phys. A 700, 359 (2002) arXiv:nucl-th/0107038.

13. D. B. Leinweber, Phys. Rev. D 53 (1996) 5115 arXiv:hep-ph/9512319.

14. J. N. Labrenz and S. R. Sharpe, Phys. Rev. D 54 (1996) 4595 arXiv:hep-lat/9605034.

15. R. D. Young et al., Phys. Rev. D 66 (2002) 094507 arXiv:hep-lat/0205017.

16. D. B. Leinweber, R. M. Woloshyn and T. Draper, Phys. Rev. D 43 (1991) 1659.

17. D. B. Leinweber et al., Phys. Rev. D 46 (1992) 3067 arXiv:hep-lat/9208025. 